\title{
Deoxygenation of Alcohols Employing Water as the Hydrogen Atom Source
}

\section{Supporting Online Material}

David A. Spiegel, Kenneth B. Wiberg Laura N. Schacherer, Matthew R. Medeiros and John L. Wood*

Sterling Chemistry Laboratory, Department of Chemistry, Yale University, New Haven, CT 06520-8107

\section{General Experimental Procedures.}

Unless stated otherwise, reactions were performed in flame-dried glassware under nitrogen atmosphere. Benzene was obtained as ACS reagent grade, and was dried in a solvent purification system (Innovative Technologies, Inc., Newburyport, MA) by passage through a $1 \mathrm{~m}$ column containing activated alumina. Water was deionized and double distilled. Trimethylborane gas was purchased from Aldrich Chemical Company (Milwaukee, WI), and dispensed through a two-stage gas regulator fitted with a Matheson Model 4775 cross-purge assembly. Trimethylborane- $\mathrm{d}_{9}$ was generated following Brown's procedure. ${ }^{1}$ Unless otherwise indicated, triethylborane and tributylborane used in these studies were purchased as the neat liquids (Aldrich Chemical Company, Milwaukee, WI), used without further purification, and stored under nitrogen atmosphere. Deuterium oxide (99.999\% isotopic purity), purchased from Cambridge Isotope Laboratories (Cambridge, MA), was used without further purification, and ampoules of this reagent were opened under nitrogen atmosphere in an Aldrich Atmos-bag (Aldrich 
Chemical Company, Milwaukee, WI) dried over calcium carbonate (drierite) and $\mathrm{P}_{2} \mathrm{O}_{5}$. All other commercially obtained reagents were used as received.

In all experiments, substrates were dried azeotropically with benzene, and air was dried by passage through concentrated sulfuric acid. O-cholesteryl-S-methyl dithiocarbonate (3), ${ }^{2}$ O-cyclododecyl-S-methyl dithiocarbonate (4), ${ }^{3}$ O-(1,2:5,6-di-Oisopropylidene- $\alpha$-D-glucofuranosyl)-S-methyl dithiocarbonate $\quad(5),{ }^{4} \quad O$-(2,3:5,6-di-Oisopropylidene- $\alpha$-D-mannofuranosyl)-S-methyl dithiocarbonate $(\mathbf{6}),^{5}$ (-)-O-menthyl-Smethyl dithiocarbonate (7), ${ }^{6}$ and $O$-dodecyl-S-methyl dithiocarbonate $(\mathbf{8})^{7}$ were all prepared according to known literature protocols. Compounds 3-7 were recrystallized between two and eight times from the following solvent systems to insure purity: $\mathbf{3}$ and $\mathbf{7}$ - EtOH; 4 - EtOH/ $\mathrm{CH}_{2} \mathrm{Cl}_{2} ; \mathbf{5}$ - petroleum ether; $\mathbf{6}$ - hexanes. Xanthate $\mathbf{8}$ was purified by flash chromatography on silica gel (gradient elution, hexanes to 10\% EtOAc in hexanes) followed by bulb-to-bulb distillation of volatile impurities.

Unless stated otherwise, all reactions were magnetically stirred and monitored by thin-layer chromatography (TLC) using E. Merck silica gel $60 \mathrm{~F}_{254}$ precoated plates $(0.25$ $\mathrm{mm}$ ). Column or flash chromatography was performed with the indicated solvents using silica gel (230-400 mesh) purchased from Bodman. All melting points were obtained on a Gallenkamp capillary melting point apparatus and are uncorrected. Infrared spectra were recorded on a Midac M1200 FTIR. ${ }^{1} \mathrm{H},{ }^{2} \mathrm{H}$, and ${ }^{13} \mathrm{C}$ NMR spectra were recorded on AM-500, Bruker Avance DPX-500 or Bruker Avance DPX-400 spectrometers. Chemical shifts are reported relative to internal chloroform $\left({ }^{1} \mathrm{H}, \delta 7.26 \mathrm{ppm} ;{ }^{13} \mathrm{C}, \delta 77.0\right.$ ppm). High resolution mass spectra were performed at the University of Illinois Mass Spectrometry Center or the University of California, Davis Mass Spectrometry Center. GC analyses were performed using a DB-1301 narrow bore column. Response factors were calculated from the ratios of pure product to added naphthalene in GC traces. 
Deuterium incorporations were determined by analysis of isotope patterns obtained either from gas chromatographic-mass spectrometric (GC/MS), or electrospray ionization (ESI) spectra. ${ }^{8-10}$ GC/MS experiments were performed using an Agilent $6890 \mathrm{~N}$ gas chromatogram equipped with an Allteck EC-5 column, coupled to an Agilent 5973N mass detector. ESI data was acquired either using Waters ZQ 4000 spectrometer, or an FTICR-MS instrument by Dr. TuKiet Lam at the W. M. Keck Foundation Biotechnology Resource Laboratory (New Haven, CT). For samples subjected to gas chromatographicmass spectrometric (GC/MS) analysis, mass spectra were obtained by averaging mass measurements over the duration of the appropriate peak in the gas chromatogram, and calculations of percent deuterium incorporation were performed on measured mass spectral peak heights, representing the average of triplicate experiments. Data obtained from ESI analysis were derived from peak integrals of the appropriate $[\mathrm{M}+\mathrm{H}]^{+}$or $[\mathrm{M}+\mathrm{Na}]^{+}$ions in a composite spectrum computed from 300 separate mass measurements.

Computational Studies. The compounds were studied using the G3 model chemistry developed by Pople, et al. ${ }^{11}$ The calculated enthalpies at $298 \mathrm{~K}$ including the zero-point energies are as follows:

$\begin{array}{lrlr}\text { Compound } & \mathrm{H}(298 \mathrm{~K}) & \text { Compound } & \mathrm{H}(298 \mathrm{~K}) \\ \mathrm{H} . & -0.50236 & \mathrm{HO} & -75.69160 \\ \mathrm{CH}_{3} . & -39.78906 & \mathrm{H}_{2} \mathrm{O} & -76.37827 \\ \mathrm{Me}_{3} \mathrm{~B}\left(\mathrm{C}_{3 \mathrm{v}}\right) & -144.42873 & \mathrm{Me}_{3} \mathrm{~B}-\mathrm{OH}_{2}\left(\mathrm{C}_{1}\right) & -220.81577 \\ \mathrm{Me}_{3} \mathrm{~B}-\mathrm{OH}\left(\mathrm{C}_{\mathrm{s}}, \mathrm{TS}\right) & -220.17602 & \mathrm{Me}_{2} \mathrm{BOH} & -180.40712 \\ \mathrm{Me}_{3} \mathrm{Al} & -361.88427 & \mathrm{Me}_{3} \mathrm{Al}-\mathrm{OH} & -438.28893 \\ \mathrm{Me}{ }_{3} \mathrm{Al}-\mathrm{OH} & -437.60210 & \mathrm{Me}_{2} \mathrm{AlOH} & -397.86650\end{array}$

Geometry optimization for the $\mathrm{Me}_{3} \mathrm{~B}-\mathrm{OH}$ radical could be effected if it were given $\mathrm{C}_{\mathrm{s}}$ symmetry, but it was found to be a transition state. Reducing the symmetry by moving 
the $\mathrm{OH}$ hydrogen and continuing the geometry optimization led to loss of a methyl radical and the formation of $\mathrm{Me}_{2} \mathrm{BOH}$.

\section{General Experimental Procedure for Deuterium Labeling Studies (Table 1).}

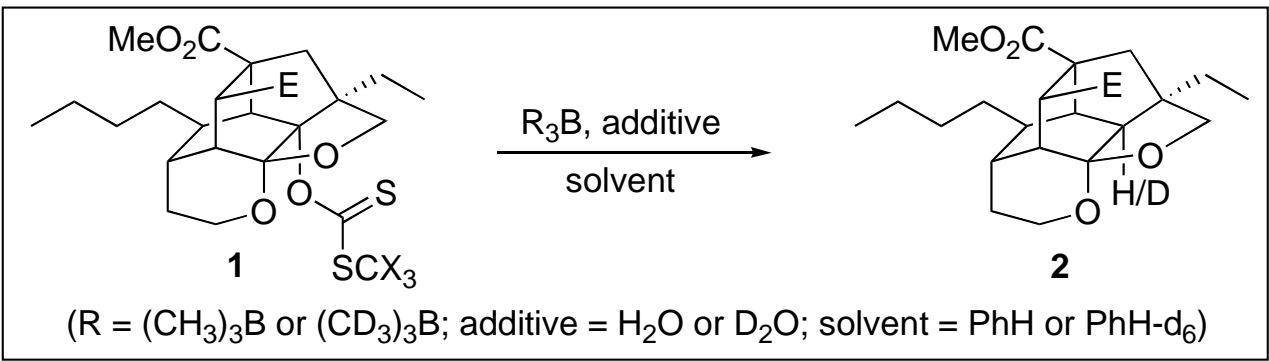

Xanthate ester $\mathbf{1}^{12}$ (3 mg, 0.006 mmol, 1.0 equiv) was dissolved in $\mathrm{PhH}$ or $\mathrm{PhH}-\mathrm{d}_{6}$ (0.2 mL) with or without additive (20 equiv) as indicated in the table. The solution was then allowed to degas by gentle purging with nitrogen for $10 \mathrm{~min}$, at which time trimethylborane or trimethylborane- $d_{9}$ (50-100 equiv) was bubbled into the reaction mixture continuously until TLC analysis indicated the complete disappearance of starting material. The solution was then purged with nitrogen over approximately $30 \mathrm{~min}$, solvent was removed in vacuo, and crude isolates were subjected either to ${ }^{1} \mathrm{H}-\mathrm{NMR}$ analysis (entries 1-3) or ${ }^{1} \mathrm{H}-\mathrm{NMR}$ followed by mass spectral analysis (entries 4-7). In all cases, Dincorporation values calculated by both methods (NMR and mass spectrometry) were found to be in good agreement.

Xanthate 1. This material was synthesized as previously described. ${ }^{12}$ For preparing the deuterated xanthate (Table $1, \mathrm{X}=\mathrm{D}$ ) an identical procedure was followed except MeI- $\mathrm{d}_{3}$ was employed in place of MeI. Compound $\mathbf{1}(\mathrm{X}=\mathrm{H})$ exhibited the following spectral characteristics: IR (thin film/NaCl) 2955 (m), 2926 (m), 2864 (w), 1731 (s), 1438 (w), 1231 (s), 1148 (m), 1093 (s), 1036 (s), 730 (w) cm ${ }^{-1} ;{ }^{1} \mathrm{H}$ NMR (500 $\left.\mathrm{MHz}, \mathrm{CDCl}_{3}\right) \delta 4.94$ (s, 1H), 3.92-3.82 (m, 3H), 3.68 (s, 3H), 3.61 (s, 3H), 3.4 (d, $J=8.5$ Hz, 1H), 3.2 (s, 1H), 2.66 (d, $J=14$ Hz, 1H), 2.55 (d, $J=1.5$ Hz, 1H), 2.53 (s, 3H), 2.19 
(d, $J=14 \mathrm{~Hz}, 1 \mathrm{H}), 2.07-1.98$ (m, 2H), 1.8-1.75 (m, 2H), 1.68-1.54 (m, 3H), 1.39-1.36 (m, 2H), 1.28-1.18 (m, 3H), 0.89-0.79 (m, 6H); ${ }^{13} \mathrm{C}$ NMR (100 MHz, $\left.\mathrm{CDCl}_{3}\right) \delta$ 213.6, 176.3, 172, 109, 103.2, 77.2, 76.4, 62, 55.8, 52.8, 51.7, 48.3, 48.3, 44.9, 41.2, 39.3, 35, 33.1, 31.1, 29.7, 28.3, 22.8, 19.7, 14, 9.3; HRMS (FAB) m/z 513.1980 [calc'd for $\mathrm{C}_{25} \mathrm{H}_{36} \mathrm{O}_{7} \mathrm{~S}_{2}(\mathrm{M}+\mathrm{H})$ 513.1983]. Deuterated Xanthate $1(\mathrm{X}=\mathrm{D})$ exhibited the following spectral characteristics: IR (thin film/NaCl) 2955 (m), 2926 (m), 2857 (w), 1730 (s), 1459 (w), 1434 (w), 1287 (w), 1231 (s), 1213 (s), 1186 (m), 1150 (m), 1124 (w), 1091 (s), 1058 (s), 1039 (s) cm ${ }^{-1}$; ${ }^{1} \mathrm{H}$ NMR (500 MHz, $\left.\mathrm{CDCl}_{3}\right) \delta 5.00$ (s, $\left.1 \mathrm{H}\right), 3.95-3.89(\mathrm{~m}, 3 \mathrm{H}$ ), 3.75 (s, 3H), 3.68 (s, 3H), 3.68 (d, $J=8.5 \mathrm{~Hz}, 1 \mathrm{H}$ ), 3.21 (s, 1H), 2.75 (d, $J=14 \mathrm{~Hz}, 1 \mathrm{H}$ ), 2.57 (d, $J=1.5 \mathrm{~Hz}, 1 \mathrm{H}), 2.42(\mathrm{~d}, J=14 \mathrm{~Hz}, 1 \mathrm{H}), 2.08-1.97(\mathrm{~m}, 2 \mathrm{H}), 1.8-1.78(\mathrm{~m}, 2 \mathrm{H})$, 1.68-1.58 (m, 3H), 1.39-1.36 (m, 2H), 1.28-1.17 (m, 3H), 0.90-0.82 (m, 6H); ${ }^{13} \mathrm{C}$ NMR (125 MHz, $\left.\mathrm{CDCl}_{3}\right) \delta$ 214.0, 176.6, 172.3, 109.4, 103.6, 77.6, 76.8, 62.5, 56,2, 53.2, 52.0, 48.7, 45.3, 42.1, 39.8, 35.5, 33.6, 31.5, 30.1, 28.6, 23.2, 23.1, 14.4, 9.6; HRMS (FAB) $\mathrm{m} / \mathrm{z}$ 516.2167 [calc'd for $\mathrm{C}_{25} \mathrm{H}_{34} \mathrm{D}_{3} \mathrm{O}_{7} \mathrm{~S}_{2}(\mathrm{M}+\mathrm{H})$ 516.2169].

Pentacycle 2. This material exhibited the following spectral characteristics: IR (thin film/NaCl) 2955 (m), 2928 (m), 2858 (m), 1728 (s), 1460 (w), 1434 (w), 1379 (w), 1282 (w), 1223 (s), 1178 (w), 1031 (m) cm ${ }^{-1}$; ${ }^{1} \mathrm{H}$ NMR (500 MHz, $\mathrm{CDCl}_{3}$ ) $\delta 3.81$ (dd, $J=$ 6, $12 \mathrm{~Hz}, 1 \mathrm{H}$ ), 3.72 (d, $J=9 \mathrm{~Hz}, 1 \mathrm{H}), 3.7$ (s, 3H), 3.65 (s, 3H), 3.39 (d, $J=9 \mathrm{~Hz}, 1 \mathrm{H}$ ), 3.14 (s, 1H), 2.7 (t, $J=3 \mathrm{~Hz}, 1 \mathrm{H}$ ), 2.67 (s, 1H), 2.37 (d, $J=3.5 \mathrm{~Hz}, 1 \mathrm{H}), 2.05$ (d, $J=14$ Hz, 1H), 1.93-1.86 (m, 1H), 1.78-1.77 (m, 1H), 1.7-1.58 (m, 2H), 1.47 (s, 1H), 1.25-1.12 (m, 8H), 0.96-0.85 (m, 6H); ${ }^{13} \mathrm{C}$ NMR (125 MHz, $\left.\mathrm{CDCl}_{3}\right) \delta$ 177.1, 172.3, 109.1, 76.8, 61.1, 57, 52.4, 53.3, 51.5, 51, 48.7, 44.7, 44.4, 39.7, 39.1, 36.8, 35.5, 31.8, 30.5, 30, 22.7, 14, 10.2; HRMS (FAB) $\mathrm{m} / \mathrm{z} 407.2432$ [calc'd for $\mathrm{C}_{23} \mathrm{H}_{34} \mathrm{O}_{6}(\mathrm{M}+\mathrm{H})$ 407.2433].

Deuterated Pentacycle 2. This material exhibited the following spectral characteristics: 2955 (m), 2926 (m), 2857 (m), 1727 (s), 1459 (w), 1434 (w), 1281 (w), 
1223 (s), 1093(s), 1023 (m) cm ${ }^{-1} ;{ }^{1} \mathrm{H}$ NMR (500 MHz, $\mathrm{CDCl}_{3}$ ) $\delta 3.81$ (dd, $J=6,12 \mathrm{~Hz}$, 1H), 3.72 (d, $J=9 \mathrm{~Hz}, 1 \mathrm{H}$ ), 3.7 (s, 3H), 3.65 (s, 3H), 3.39 (d, $J=9 \mathrm{~Hz}, 1 \mathrm{H}), 3.14$ (s, 1H), 2.7 (t, $J=3 \mathrm{~Hz}, 1 \mathrm{H}), 2.67$ (s, 1H), 2.37 (d, $J=3.5 \mathrm{~Hz}, 1 \mathrm{H}), 2.05$ (d, $J=14 \mathrm{~Hz}, 1 \mathrm{H}), 1.93-$ $1.86(\mathrm{~m}, 1 \mathrm{H}), 1.78-1.77$ (m, 1H), 1.7-1.58 (m, 2H), 1.47 (s, 1H), 1.25-1.12 (m, 8H), 0.960.85 (m, 6H); ${ }^{13} \mathrm{C}$ NMR (100 MHz, PhH-d 6 ) $\delta$ 177.4, 172.6, 109.8, 77.8, 61.1, 52.9, 52.3, 51.9, 51.6, 49.6, 45.5, 45.2, 40.8, 39.7, 37.7, 36.3, 32.6, 31.1, 30.8, 23.7, 17.7, 14.7, 10.2; HRMS (ESI) $\mathrm{m} / \mathrm{z} 408.2498$ [calc'd for $\mathrm{C}_{23} \mathrm{H}_{33} \mathrm{DO}_{6}(\mathrm{M}+\mathrm{H}) 408.2491$ ].

\section{General Procedure for $\mathrm{BMe}_{3}$-Mediated Xanthate Deoxygenation Reactions (Table}

2). A solution of xanthate (95-150 mg, 0.15-0.35 mmol, 1.0 equiv) dissolved in benzene (0.03 M) was degassed by bubbling with argon gas for $1 \mathrm{~h} . \mathrm{BMe}_{3}$ gas (2.5-4.0 equiv) was then bubbled through the solution over 30-60 min. After $\mathrm{BMe}_{3}$ addition was complete, air ( 0.8 equiv of $\mathrm{O}_{2}$ ) was introduced into reactions via a cannula placed beneath the solvent surface at a rate of $1.25 \mathrm{~mL} / \mathrm{hr}$, unless otherwise indicated. At this point, argon gas was bubbled vigorously through the reaction mixture for a period of 1 hour, followed by distillation of the remaining volatiles via rotary evaporation. When necessary, reaction products were then further purified chromatographically on silica gel.

The only observable reagent-derived byproduct in ${ }^{1} \mathrm{H}-\mathrm{NMR}$ and GC-MS spectra of $\mathrm{BMe}_{3}$-mediated reductions was S,S-dimethyl dithiocarbonate. A sample of this material (210 mg, obtained from TCI America, Portland, OR) was evaporated to dryness after co-distillation with benzene ( $25 \mathrm{~mL}$ ) followed placement of the residue under vacuum for 30 minutes. Occasionally however, reaction residues obtained following solvent removal, while consisting exclusively of reduction products by ${ }^{1} \mathrm{H}-\mathrm{NMR}$ spectroscopy, persisted in emitting a thiol odor. Filtration of these isolates through silica removed these odorous impurities in all cases. 
General Procedure for $\mathbf{B E t}_{3}$ or $\mathbf{B B u}_{3}$-Mediated Reactions (Table 3). These reactions were performed under conditions identical with those reported above for $\mathrm{BMe}_{3}$, however $\mathrm{BEt}_{3}$ or $\mathrm{BBu}_{3}$ (4.0 equiv diluted to $1 \mathrm{~mL}$ in $\mathrm{PhH}$ ) was added via syringe over a period of 30-60 min in place of $\mathrm{BMe}_{3}$. Upon completion of air addition, a $3 \mathrm{M}$ solution of aqueous sodium hydroxide and $30 \%$ aqueous hydrogen peroxide were added slowly to reaction mixtures (CAUTION: reaction is exothermic). After stirring for 10-30 min, the resulting biphasic mixture was diluted with hexanes (for reactions with xanthates $\mathbf{1 , 5}$ and $\mathbf{6}$ ) or ethyl acetate (for reactions with xanthates $3,4,7$, and $\mathbf{8}$ ), and the layers were separated. The organic layer was then washed with brine, and combined aqueous layers back extracted with either hexanes or ethyl acetate. Organic extracts were dried over sodium sulfate, filtered, and concentrated under reduced pressure. When necessary, reaction products were further purified chromatographically on silica gel.

\section{Sample Experimental Procedures.}

\section{Preparation of 1-deoxy-2,3:5,6-di- $O$-isopropylidene- $\alpha$-D-mannofuranose.}

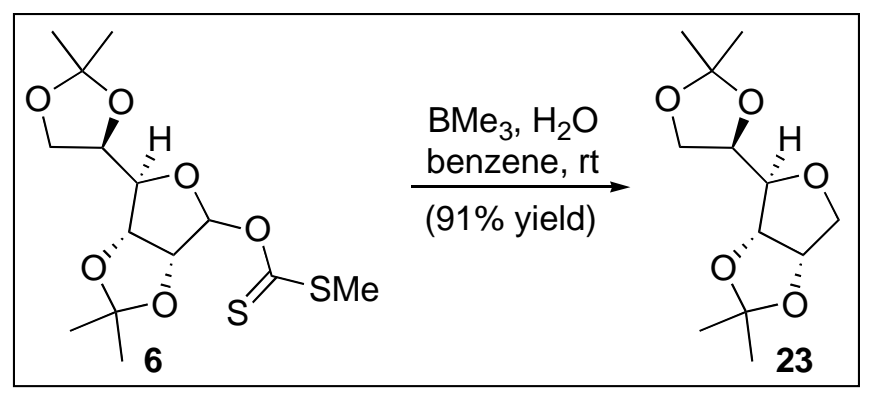

1-deoxy-2,3:5,6-di-O-isopropylidene- $\alpha$-D-mannofuranose. Methyl xanthate 6 (97 mg, $0.28 \mathrm{mmol}, 1.0$ equiv) was transferred to a flame-dried $100 \mathrm{~mL}$ pear-shaped flask, dried azeotropically with benzene ( $5 \mathrm{~mL} x$ 3), and held under vacuum for $2 \mathrm{~h}$. The residue was then redissolved in benzene (9.2 mL), combined with $\mathrm{H}_{2} \mathrm{O}(25 \mu \mathrm{L}$, $1.38 \mathrm{mmol}$, 5.0 equiv) and allowed to stir for $1 \mathrm{~h}$. Argon gas was then bubbled through the solution for a period 
of $1 \mathrm{~h}$, after which, $\mathrm{BMe}_{3}$ gas (1.1 mmol, 4.0 equiv) was introduced over $0.5 \mathrm{~h}$ via a cannula placed underneath the reaction surface. At this time, air (27 mL, $0.22 \mathrm{mmol}, 0.8$ equiv) was introduced via syringe pump addition over approximately $21 \mathrm{~h}$, the reaction mixture was purged with argon for $1 \mathrm{~h}$ and concentrated under reduced pressure. Filtration over a plug of silica gel (10\% EtOAc in hexanes, eluent) provided 23 as a pale yellow oil (61 mg, 91\% yield), which solidified upon storage at reduced temperature. ${ }^{13}$

\section{Preparation of deuterocyclododecane using $\mathrm{BBu}_{3}$.}

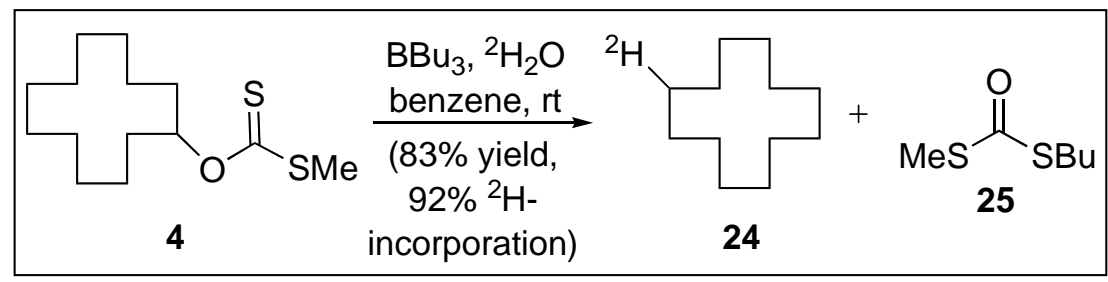

Deuterocyclododecane. To a solution of $O$-cyclododecyl $S$-methyl dithiocarbonate (1, $145 \mathrm{mg}, 0.53 \mathrm{mmol}, 1.0$ equiv) dissolved in dry benzene $(17 \mathrm{~mL})$ was added $\mathrm{D}_{2} \mathrm{O}(52 \mu \mathrm{L}$, $2.6 \mathrm{mmol}, 5.0$ equiv) and the mixture was allowed to stir for 1 hour. Argon gas was then bubbled through the solution for a period of $1 \mathrm{~h}$, at which time, a solution of $\mathrm{BBu}_{3}(0.65$ $\mathrm{mL}, 2.65 \mathrm{mmol}, 5.0$ equiv) in benzene $(0.35 \mathrm{~mL})$ was added via syringe pump over 25 min. On completion of $\mathrm{BBu}_{3}$ addition, dry air (52 $\mathrm{mL}, 0.42 \mathrm{mmol} \mathrm{O}, 0.8$ equiv) was introduced via syringe pump at a rate of $1.2 \mathrm{ml} / \mathrm{hr}$ through a stainless steel cannula positioned beneath the reaction surface. At this time, hydrogen peroxide (30\% aqueous solution, $5 \mathrm{~mL}$ ) and sodium hydroxide (3 $\mathrm{M}$ aqueous solution, $5 \mathrm{~mL}$ ) were introduced simultaneously into the reaction mixture (CAUTION: reaction is exothermic). After stirring for $10 \mathrm{~min}$, the solution was diluted with brine $(10 \mathrm{~mL})$ and EtOAc $(10 \mathrm{~mL})$. The organic phase was then separated and the aqueous layer was extracted with EtOAc (10 mL x 3). Combined organic portions were dried over anhydrous sodium sulfate, filtered, and solvent evaporated under reduced pressure to afford a clear oil. Chromatography 
through a plug of silica (gradient elution, 0 to $20 \%$ EtOAc in hexanes) provided deuterocyclododecane (24, $74 \mathrm{mg}, \mathbf{8 3 \%}$ yield, 92\% ${ }^{2} \mathrm{H}$-incorporation) as a white crystalline solid; mp 49-51 ${ }^{\circ}$ (hexanes); ${ }^{1} \mathrm{H}$ NMR (500 MHz, $\left.\mathrm{CDCl}_{3}\right) \delta 1.34(\mathrm{~s}, 24 \mathrm{H}) ;{ }^{2} \mathrm{H}$ NMR (500 MHz, $\left.\mathrm{CHCl}_{3}\right) \delta 1.33$; LRMS (EI) $\mathrm{m} / \mathrm{z} 169.1$ [calc'd for $\mathrm{C}_{12} \mathrm{H}_{23} \mathrm{D}(\mathrm{M}+\mathrm{H})$ 169.1] and $S$-methyl-S-butyl dithiocarbonate (25, $84 \mathrm{mg}, 97 \%$ yield). $\boldsymbol{S}$-methyl-S-butyl dithiocarbonate (25). This material was isolated as a pale yellow oil with the following spectral characteristics: IR (thin film/NaCl) 2957 (s), 2931 (s), 2871 (m), 1740 (w), 1648 (s), 1465 (w), 867 (s) cm ${ }^{-1} ;{ }^{1} \mathrm{H}$ NMR (500 MHz, $\mathrm{CDCl}_{3}$ ) $\delta 2.99$ (t, J = $7 \mathrm{~Hz}, 2 \mathrm{H}$ ), 2.41 (s, 3H), 1.62-1.56 (m, 2H), 1.42-1.34 (m, 2H), 0.90 (t, $J=7.5 \mathrm{~Hz}, 3 \mathrm{H}) ;{ }^{13} \mathrm{C}$ NMR (125 MHz, $\left.\mathrm{CDCl}_{3}\right) \delta 190.0,31.8,30.4,21.8,13.5,13.0$.

\section{Characterization Data for Reduction Products.}

Following the general procedures outlined above, the known materials listed below were isolated in the yields indicated in Tables 2-3. Structure assignments were based upon comparison to available literature data for the following materials: cholest-5ene, ${ }^{14} \quad 3$-deutero-cholest-5-ene, ${ }^{15} \quad 3$-deoxy-1,2:5,6-di-O-isopropylidene- $\alpha$-Dglucofuranose $^{16}$ 3-deoxy-3-deutero-1,2:5,6-di-O-isopropylidene- $\alpha$-D-glucofuranose, ${ }^{17}$ and 1-deoxy-2,3:5,6-di-O-isopropylidene- $\alpha$-D-mannofuranose. ${ }^{13}$ An authentic sample of cyclododecane was purchased from TCI America (Portland, OR),

1-deoxy-1-deutero-2,3:5,6-di- $O$-isopropylidene- $\alpha$-D-mannofuranose. This material was isolated as an amorphous white solid in the yields indicated in Tables 2-3, following the general procedures outlined above. Structure assignment was based upon comparison with the known compound 1-deoxy-2,3:5,6-di-O-isopropylidene- $\alpha$-Dmannofuranose, ${ }^{13}$ as well as the following spectral data: IR (thin film/ $\mathrm{NaCl}$ ) 2987 (s), 
2939 (m), 2876 (w), 2143 (w), 1379 (s), 1372 (s), 1258 (s), 1210 (s), 1162 (s), 1119 (s), 1067 (s), 851 (s) cm ${ }^{-1} ;{ }^{1} \mathrm{H}$ NMR (500 MHz, $\mathrm{CDCl}_{3}$ ) $\delta$ 4.80-4.73 (m, 2H), 4.43-4.40 (m, 1H), 4.11-4.01 (m, 3H), 3.49-3.47 (m, 1H), 1.49 (s, 3H), 1.45 (m, 3H), 1.38 (s, 3H), 1.34 (s, 3H); ${ }^{13} \mathrm{C}$ NMR $\left(125 \mathrm{MHz}, \mathrm{CDCl}_{3}\right) \delta$ 112.2, 108.9, 82.7, 80.8, 80.3, 73.2, 73.7 (t), 66.7, 26.8, 25.8, 25.2, 24.4. LRMS (ESI) $\mathrm{m} / \mathrm{z} 268.2$ [calc'd for $\mathrm{C}_{12} \mathrm{H}_{19} \mathrm{DO}_{5}(\mathrm{M}+\mathrm{Na})$ 268.2], 93\% D-incorporation.

\section{References.}

\section{Spectral Data.}

Spectral Data for Xanthate $1(X=D)$.

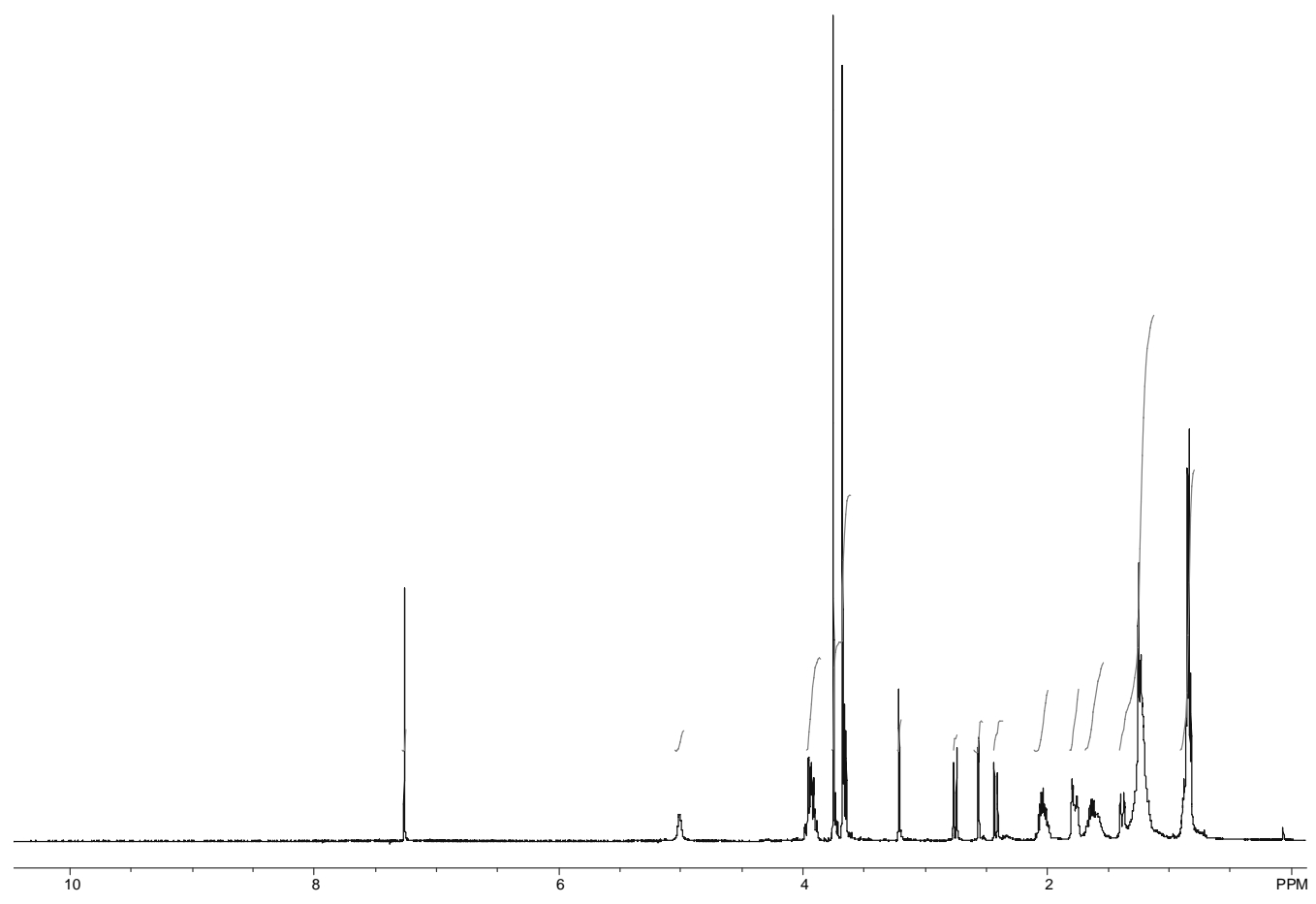

${ }^{1} \mathrm{H}$ NMR (500 MHz, $\mathrm{CDCl}_{3}$ ) of compound $1(\mathrm{X}=\mathrm{D})$. 


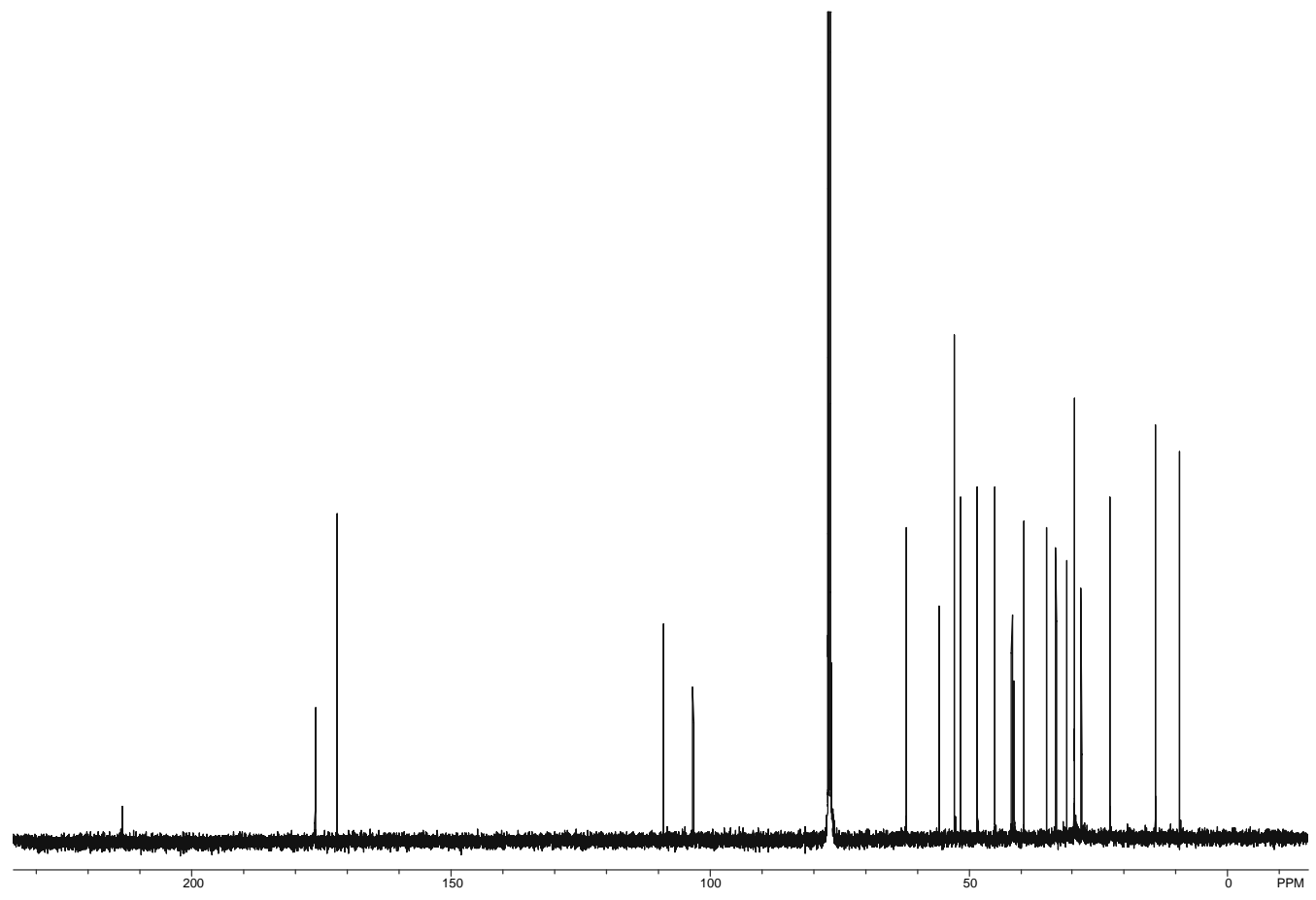

${ }^{13} \mathrm{C}$ NMR $\left(125 \mathrm{MHz}, \mathrm{CDCl}_{3}\right)$ of compound $\mathbf{1}(\mathrm{X}=\mathrm{D})$.

Spectral Data for Polycycle $2(X=D)$.

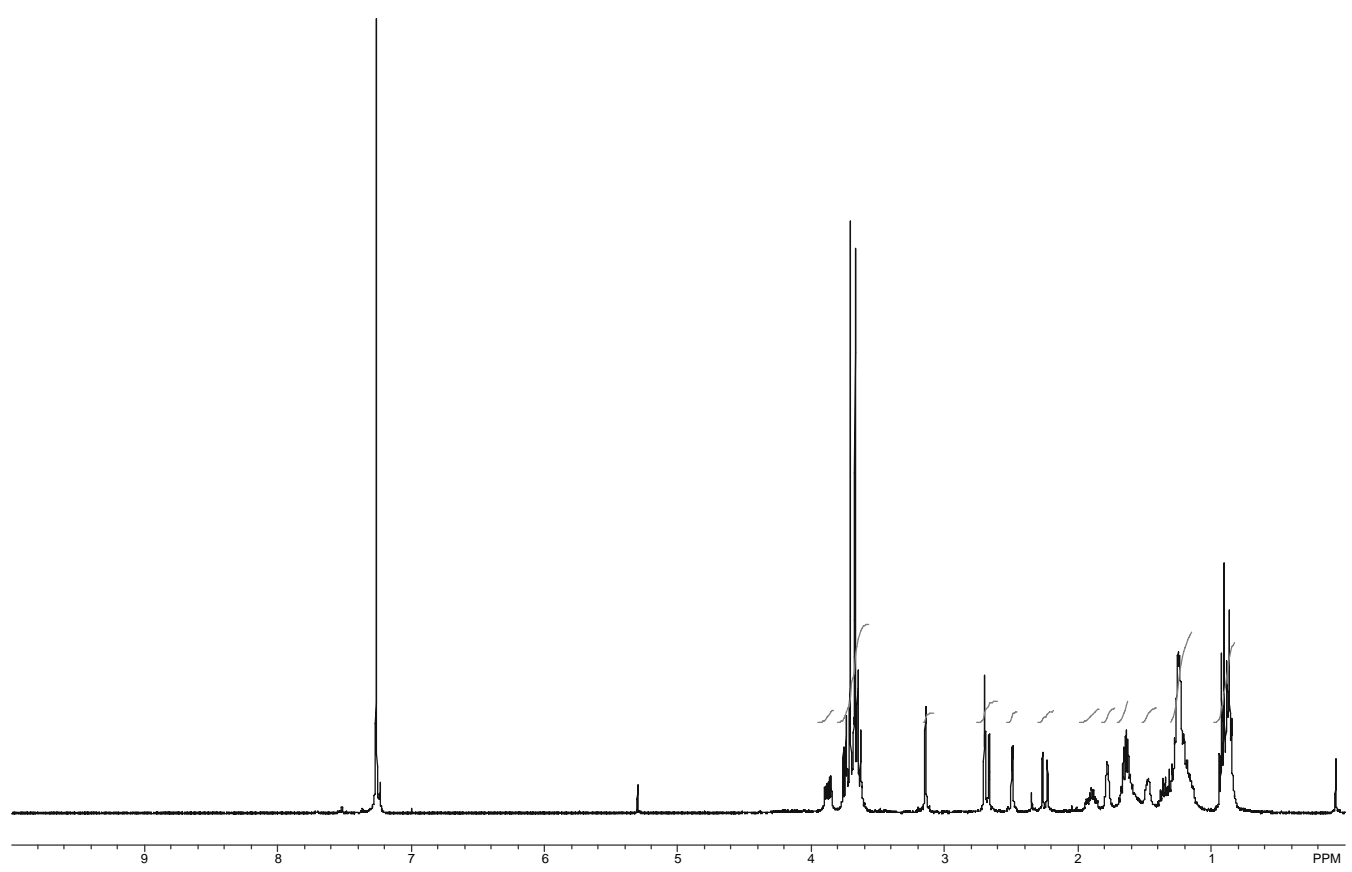

${ }^{1} \mathrm{H}$ NMR (500 MHz, $\left.\mathrm{CDCl}_{3}\right)$ of compound $2(\mathrm{X}=\mathrm{D})$. 


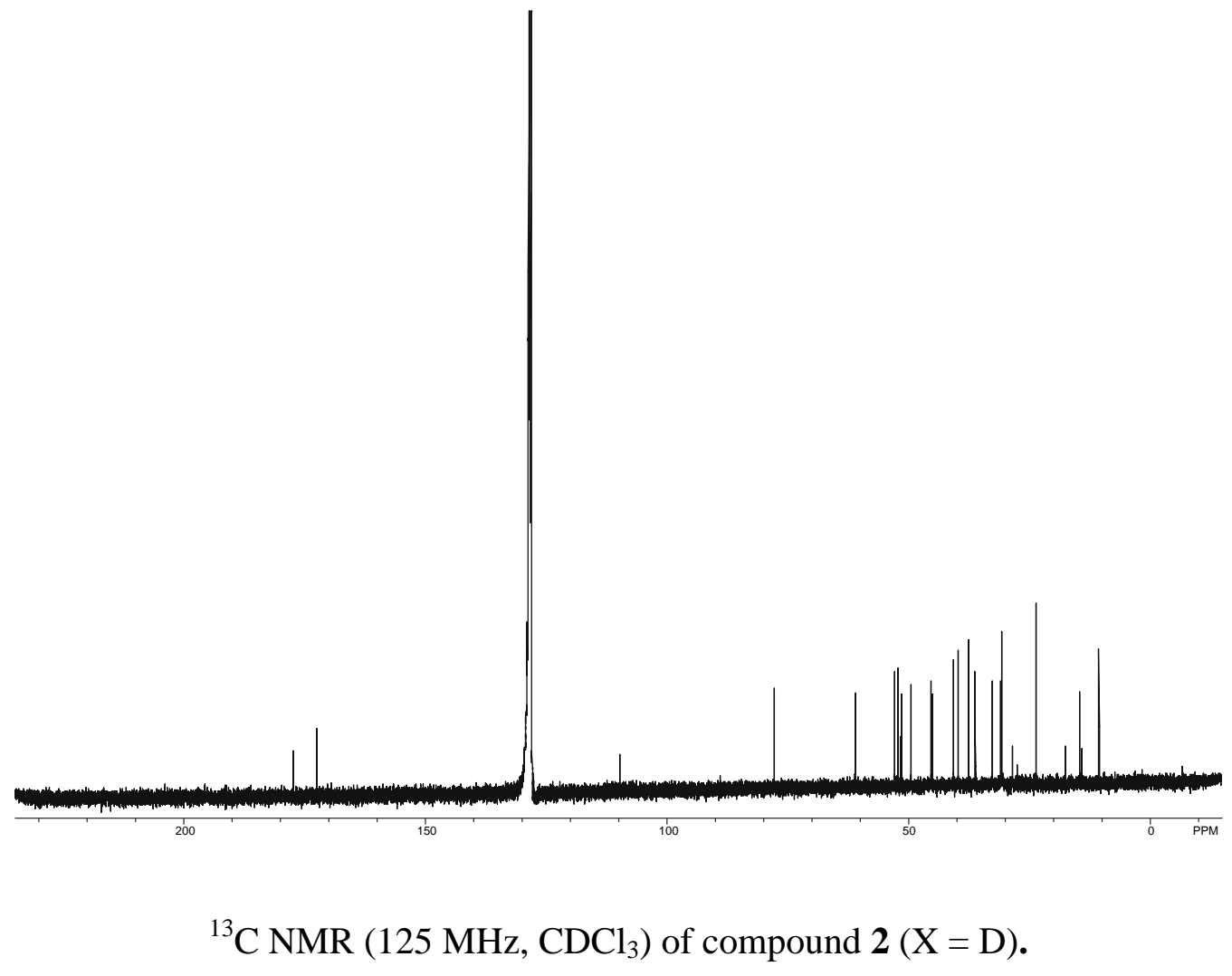

\section{Spectral Data for $S$-methyl-S-butyl dithiocarbonate.}

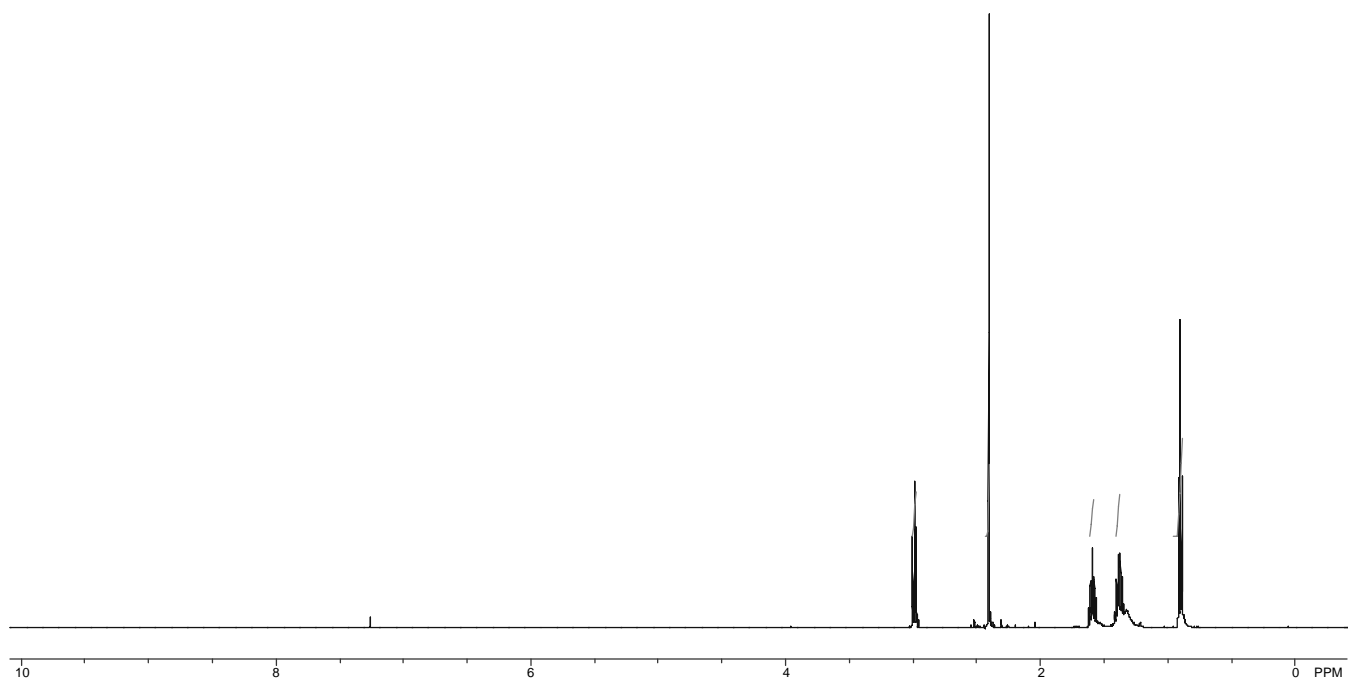

${ }^{1} \mathrm{H}$ NMR (500 MHz, $\mathrm{CDCl}_{3}$ ) of $S$-methyl-S-butyl dithiocarbonate. 


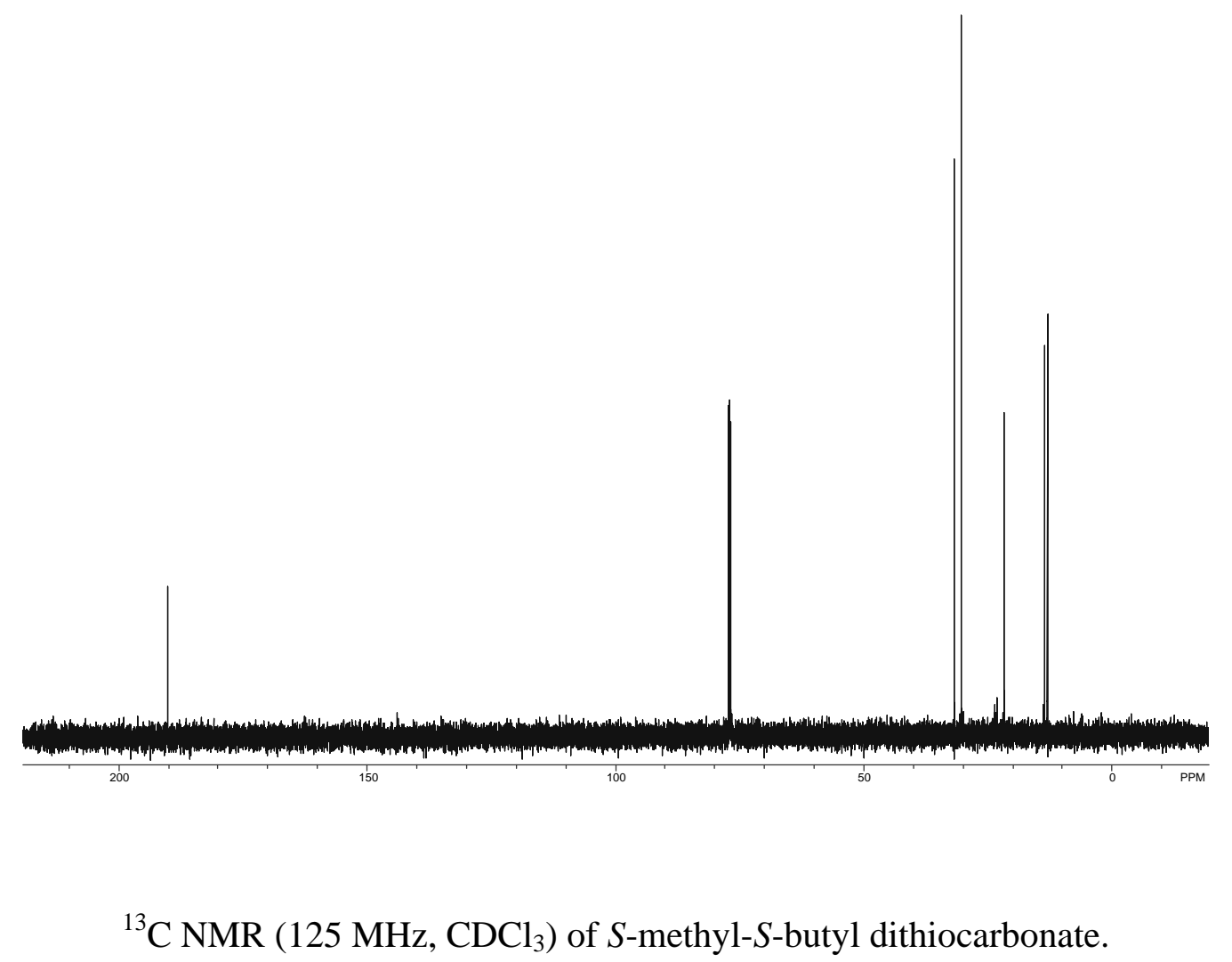

\section{References.}

(1) Brown, H. C.; Racherla, U. S. J. Org. Chem. 1986, 51, 427.

(2) Barton, D. H. R.; McCombie, S. W. J. Chem. Soc. - Perkin 1 1975, 1574.

(3) Barton, D. H. R.; Jang, D. O.; Jaszberenyi, J. C. J. Org. Chem. 1993, 58, 6838.

(4) Freudenberg, K.; Wolf, A. Chem. Ber. 1927, 60, 232.

(5) Cesare, P.; Gross, B. Synthesis 1980, 9, 714.

(6) Villemin, D.; Hachemi, M. Synth. Comm. 1996, 26, 2449.

(7) Lee, A. W. M.; Chan, W. H.; Wong, H. C.; Wong, M. S. Synth. Comm. 1989, 19, 547.

(8) Aron, Z. D.; Overman, L. E. J. Am. Chem. Soc. 2005, 127, 3380.

(9) Katta, V.; Chait, B. T. J. Am. Chem. Soc. 1993, 115, 6317.

(10) Seto, H.; Mizukai, K.; Fujioka, S.; Koshino, H.; Yoshida, S. J. Chem. Soc. Perkin 1 2002, 2395.

(11) Curtiss, L. A.; Raghavachari, K.; Reforn, P. C.; Rassolov, V.; Pople, J. A. J. Chem. Phys. 1998, 109, 7764.

(12) Njardarson, J. T.; McDonald, I. M.; Spiegel, D. A.; Inoue, M.; Wood, J. L. Org. Lett. 2001, 3, 2435.

(13) Simon, P.; Ziegler, J.-C.; Gross, B. Synthesis 1979, 951.

(14) Taylor, E. I.; Djerassi, C. J. Am. Chem. Soc. 1976, 98, 2275.

(15) Oba, M.; Nishiyama, K. Synthesis 1994, 6, 624.

(16) Tormo, J.; Fu, G. C. Org. Syn. 2001, 78, 239.

(17) Oba, M.; Nishiyama, K. Tetrahedron 1994, 50, 10193. 